\title{
Weak Massive Gravity
}

\author{
D. Comelli ${ }^{a}$, F. Nesti ${ }^{b}$ and L. Pilo ${ }^{b, c}$ \\ ${ }^{a}$ INFN - Sezione di Ferrara, I-35131 Ferrara, Italy \\ ${ }^{b}$ Dipartimento di Fisica, Università di L'Aquila, I-67010 L'Aquila \\ ${ }^{c}$ INFN, Laboratori Nazionali del Gran Sasso, I-67010 Assergi, Italy
}

(Dated: August 12, 2018)

\begin{abstract}
We find a new class of theories of massive gravity with five propagating degrees of freedom where only rotations are preserved. Our results are based on a non-perturbative and background-independent Hamiltonian analysis. In these theories the weak field approximation is well behaved and the static gravitational potential is typically screened à la Yukawa at large distances, while at short distances no vDVZ discontinuity is found and there is no need to rely on nonlinear effects to pass the solar system tests. The effective field theory analysis shows that the ultraviolet cutoff is $\left(m M_{P l}\right)^{1 / 2} \simeq$ $1 / \mu \mathrm{m}$, the highest possible. Thus, these theories can be studied in weak-field regime at all the phenomenologically interesting scales, and are candidates for a calculable large-distance modified gravity.
\end{abstract}

Introduction. The long standing quest for a healthy modification of gravity at large distances has recently been the target of renewed interest. Since the work of Fierz and Pauli [1] (FP) it was realized that in generic Lorentz-invariant (LI) massive deformations of gravity 6 DoF are present already at linearized level around Minkowski space, the sixth mode being a ghost. While a tuning can get rid of this mode at linear level, it reappears at the nonlinear level or around non-flat backgrounds [2]. It is remarkable that only recently a nonlinear completion was found which propagates five DoF also in general. This was proposed first up to the fourth order in perturbation theory [3] and then to the full nonlinear level in [4, 5]. At linearized level this theory reduces to the Lorentz-invariant FP theory, and shares with it the failure to reproduce the correct light bending, at odds with General Relativity (GR) and observations. The issue does not disappear in the limit of vanishing graviton mass, the so called vDVZ discontinuity [6]. A way out was proposed by Vainshtein in 7], by arguing that nonlinear effects restore the correct GR behavior at short distances from a source. While this mechanism has been verified in a number of models [8], the theory has to rely on strong nonlinearities even at the macroscopic solar system scales, where the gravitational potential is small, as discussed for generic LI theories in 9].

A different perspective is provided by more general theories of massive gravity where Lorentz invariance in the gravitational sector is not imposed, though the weak equivalence principle still holds. At the quadratic level a number of such theories that are free from the vDVZ discontinuity is on the market [10 14]. Moreover, in some of them the cutoff beyond which the theory cannot be trusted or where nonlinearities outbreak, has been argued to be the highest possible [10]. Clearly, if there exists nonlinear completions of such theories, they represent serious candidates for a consistent and calculable large distance modifications of gravity.

In this Letter we uncover a full class of nonlinear theories implementing these ideas. The analysis is based on a recent work [15] stating the general conditions for the propagation of five DoF in massive gravity, whose main result is reviewed in the next section. In the following section we provide the general solution to these conditions. In the remaining part we describe an explicit example providing massive gravity theories which are free from the vDVZ discontinuity at short distances, lead to screened (Yukawa) gravitational potential at large distances, propagate five massive DoF both at linear and nonlinear level, and are calculable in the weak-field limit at phenomenologically interesting distances.

Massive gravities with 5 DoF. The generic Lagrangian for massive gravity is obtained by adding a nonderivative potential $V$ for the metric $g_{\mu \nu}$,

$$
L=M_{\mathrm{pl}}^{2} \int d^{3} x \sqrt{g}\left[R-m^{2} V(g)+\mathcal{L}_{\text {matter }}\right],
$$

and its propagating DoF were analyzed in [15] using the Hamiltonian formalism, with the standard ADM decomposition [16] in terms of lapse $N$, shifts $N^{i}$ and spatial metric $\gamma_{i j}$

$$
g_{\mu \nu}=\left(\begin{array}{cc}
-N^{2}+N^{i} N^{j} \gamma_{i j} & \gamma_{i j} N^{j} \\
\gamma_{i j} N^{j} & \gamma_{i j}
\end{array}\right)
$$

The potential $V$ is regarded as a function of these variables, and it is useful to define $\mathcal{V} \equiv m^{2} N \operatorname{det}(\gamma)^{1 / 2} V$. We will also denote $N^{A}=\left(N, N^{i}\right)$ with $A=0,1,2,3$ (note $N^{A}$ is not a Lorentz 4 -vector), and with $F_{A B \ldots}$ the derivative of any $F$ with respect to $N^{A}, N^{B}$, etc.

As it stands, the presence of $V$ in the action violates diffeomorphism invariance, and in principle six modes can propagate, with a (sixth) mode being typically a ghost. The conditions on the potential $\mathcal{V}\left(N^{A}, \gamma^{i j}\right)$ to have 5 propagating DoF and unbroken rotations were found in [15], and they turned out to be quite simple:

- First, the four secondary constraints have to determine only three out of four auxiliary fields [5, 15], leaving a new constraint. For this, the hessian of the potential with respect to lapse and shifts must have rank 3

$$
\operatorname{det} \mathcal{V}_{A B}=0, \quad\left(\operatorname{rank} \mathcal{V}_{A B}=3\right)
$$

So, $\mathcal{V}_{A B}$ has only one null eigenvector $\chi^{A}: \mathcal{V}_{A B} \chi^{B}=0$. 
- Second, the tertiary constraint has to be free of the last auxiliary field [15], to complete the new constraint in phase space. This amounts to the following additional condition

$$
\tilde{\mathcal{V}}_{i}+2 \xi^{A} \xi^{j} \frac{\partial \tilde{\mathcal{V}}_{A}}{\partial \gamma^{i j}}=0
$$

Here we defined $\tilde{\mathcal{V}}=\gamma^{-1 / 2} \mathcal{V}$ and $\xi^{A}=\chi^{A} / \chi^{0}$. We are free to normalize to $\chi^{0}=1$.

It can be shown that the same eqs. (3), (4) hold also for explicitly time dependent potentials [18]. One can verify that the Lorentz-invariant massive gravity theory of [4, 5] nontrivially satisfies both (3) and (4). However, we show here that a wider class of solutions with phenomenologically interesting features exist when the potential is not Lorentz invariant but only rotational invariant. To this aim, let us the discuss the general solution to (3) and (4).

General solution. We start from eq. (3), by recalling that it is a 'homogeneous Monge-Ampere' equation, a well studied problem in mathematical physics. In the very clear work 19], the general implicit solution was found by changing variables from $N^{A}$ to the eigenvector $\chi^{A}$. In these variables, one finds that the potential must be an homogeneous function of $\chi^{A}$ of degree zero. Then the key role is played by a generic function $\mathcal{U}\left(\xi^{i}, \gamma_{i j}\right)$, defined as $\mathcal{U}=\chi^{A} \tilde{\mathcal{V}}_{A}$ and linked to $\tilde{\mathcal{V}}_{0}$ by a Legendre transformation

$$
\tilde{\mathcal{V}}_{0}=\mathcal{U}-\mathcal{U}_{i} \xi^{i}, \quad \tilde{\mathcal{V}}_{i}=\mathcal{U}_{i}
$$

Note that $\tilde{\mathcal{V}}_{A}=\partial \tilde{\mathcal{V}} / \partial N^{A}$ while $\mathcal{U}_{i}=\partial \mathcal{U} / \partial \xi^{i}$. Any function $\mathcal{U}$ will lead to a solution of the Monge-Ampere equation (3), provided (5) can be integrated to find $\mathcal{V}$. Integrability is ensured [19] by an important implicit relation between $N^{i}, N$ and $\xi^{i} 1$ which includes a new generic function $\mathcal{L}\left(\xi^{i}, \gamma^{i j}\right)$ :

$$
N^{i}-N \xi^{i}=\mathcal{U}^{i j} \mathcal{L}_{j},
$$

where $\mathcal{U}^{i j} \equiv\left(\mathcal{U}_{i j}\right)^{-1}$ (inverse of the hessian matrix) and $\mathcal{L}_{i} \equiv \partial_{\xi^{i}} \mathcal{L}$. For any $\mathcal{L}$, one should formally invert (6) to determine $\xi^{i}\left(N^{A}, \gamma^{i j}\right)$, and then integrate (5) in $N^{0}, N^{i}$ to find the potential. As a result, the general solution of (3) is expressed in terms of two generic functions $\mathcal{U}$ and $\mathcal{L}$ of $\xi^{i}$ and $\gamma^{i j}$. Note that up to now the variables $\gamma_{i j}$ inside $\mathcal{U}$ and $\mathcal{L}$ are spectators.

Equation (4) brings $\gamma_{i j}$ into the game. When written in terms of $\mathcal{U}$, it reads

$$
\frac{\partial \mathcal{U}}{\partial \xi^{i}}+2 \xi^{j} \frac{\partial \mathcal{U}}{\partial \gamma^{i j}}=0
$$

\footnotetext{
${ }^{1}$ We note en passant that this implicit change of variables between $N^{i}$ and $\xi^{i}$ trivializes to a good extent the complicated Hamiltonian analysis of [15], and contain the shift transformations of [4]. This points to a possible important physical role of the $\xi^{i}$ variables, which in ADM language are linked to a 'spatial over temporal' speed of the foliation.
}

which can be now readily solved by stating that $\mathcal{U}$ is a scalar function of a particular combination of $\xi^{i}$ and $\gamma^{i j}$ :

$$
\mathcal{U}=\mathcal{U}\left(X^{i j}\right) \quad \text { with } \quad X^{i j}=\left(\gamma^{i j}-\xi^{i} \xi^{j}\right),
$$

The last step is to find the potential by integrating the relation (5). These equations admit, as integration constant, a generic function $\mathcal{C}\left(\gamma^{i j}\right)$ to be added to $\tilde{\mathcal{V}}$.

Summarizing, we have found a whole new class of solutions propagating five DoF, which are parametrized by three generic functions $\mathcal{U}\left(X^{i j}\right), \mathcal{L}\left(\xi^{i}, \gamma^{i j}\right)$ and $\mathcal{C}\left(\gamma^{i j}\right)$. In view of unbroken rotations, $\mathcal{U}$ has to be a scalar function of $X^{i k} \delta_{k j}$, i.e. of its three invariant traces, and similarly for $\mathcal{C} \equiv \mathcal{C}\left(\gamma^{i k} \delta_{k j}\right)$.

In order to integrate (5D) and find a potential $\mathcal{V}$ explicitly, some concrete $\mathcal{L}$ has to be chosen first, to solve the implicit relation (6) between $\xi^{i}$ and $N^{A}$. We remark that in general the resulting potential, function of $N, N^{i}$ and $\gamma_{i j}$, will break Lorentz symmetry. The conditions for Lorentz invariance, leading to the potentials of [4, 5], consist in complicated additional constraints that will not be discussed here. Instead, interesting new solutions can be found by many choices of $\mathcal{L}$. The general analysis will be reported in a separate publication [18] - here, it is enough to consider the case $\mathcal{L}=0$, which already leads to interesting features and phenomenology.

An example of explicit theories. For $\mathcal{L}=0$, eq. (6) leads to the simple relation $\xi^{i}=N^{i} / N$. This gives $X^{i j}=\left(\gamma^{i j}-N^{i} N^{j^{\prime}} / N^{2}\right) \equiv g^{i j}$, which remarkably coincides with the spatial part of the inverse metric. It is pleasing that also equations (5) can be integrated and one finds the following large family of explicit potentials

$$
V=\mathcal{U}\left(g^{i k} \delta_{k j}\right)+N^{-1} \mathcal{C}\left(\gamma^{i k} \delta_{k j}\right) .
$$

It is interesting that in this example, a closed form for the Hamiltonian of the system upon using the secondary constraints can be found. Using the expressions in [15], we find that $\mathcal{U}$ cancels out and (suppressing boundary terms)

$$
H=M_{P}^{2} m^{2} \int \mathrm{d}^{3} x \sqrt{\gamma} \mathcal{C} .
$$

As a result, by choosing $\mathcal{C}$ a positive function, the Hamiltonian is positive definite.

A basic test of the above theories consists in analyzing the weak field expansion around flat space, to see whether it is healthy and the phenomenological implications are consistent with the weak field tests of gravity.

The conditions on the potential (9), to admit Flat Minkowski background $\eta_{\mu \nu}$ are

$$
\left.\mathcal{U}\right|_{g=\eta}=0, \quad\left[\mathcal{U}^{\prime}+\mathcal{C}^{\prime}-\frac{\mathcal{C}}{2}\right]_{\mid g=\eta}=0,
$$

where $\left.\right|_{g=\eta} \equiv\left\{N \rightarrow 1, N^{i} \rightarrow 0, \gamma_{i j} \rightarrow \delta_{i j}\right\}$ and $\partial_{\gamma^{i j}} \mathcal{C} \equiv$ $\mathcal{C}^{\prime} \delta_{i j}, \partial_{\gamma^{i j}} \mathcal{U} \equiv \mathcal{U}^{\prime} \delta_{i j}$ on Minkowski. 
At quadratic level, the expansion of the potential leads to mass terms for the metric fluctuations $h_{\mu \nu}=g_{\mu \nu}-\eta_{\mu \nu}$, which can be parametrized as

$$
\frac{M_{\mathrm{pl}}^{2}}{2}\left(m_{0}^{2} h_{00}^{2}+2 m_{1}^{2} h_{0 i}^{2}-m_{2}^{2} h_{i j}^{2}+m_{3}^{2} h^{2}-2 m_{4}^{2} h_{00} h\right)
$$

with $h=h_{i i}$ and repeated spatial indices are summed. The quadratic expansion reflects the breaking of Lorentz invariance in the full potential and gives the most general rotationally invariant mass terms for the graviton 2 They were studied in 10, 11], where it was shown that absence of ghosts and stability of the linearized theory can be achieved for $m_{0}=0$, together with the conditions $m_{2}^{2}>0$ and $m_{1}^{2}>m_{4}^{2}>0$. It is thus crucial to verify whether the potential (9) can satisfy these criteria. Expanding the potential (9), we get the mass spectrum

$$
\begin{array}{ll}
m_{0}^{2}=0, & m_{2}^{2}=\left.\mathcal{O}_{i j, i j}\right|_{g=\eta} \\
m_{1}^{2}=2 m_{4}^{2}=\left.3 \mathcal{U}^{\prime}\right|_{g=\eta}, & m_{3}^{2}=-\left.\mathcal{O}_{i i, j j}\right|_{g=\eta} \\
\mathcal{O}_{i_{1} j_{1}, i_{2} j_{2}} \equiv \partial_{\gamma_{i_{2} j_{2}}}\left(\partial_{\gamma_{i_{1} j_{1}}} \mathcal{V}-\frac{1}{2} \gamma_{i_{1} j_{1}} \mathcal{V}\right) .
\end{array}
$$

We thus find a strong correlation between $m_{1}$ and $m_{4}$, and their ratio $m_{1}^{2} / m_{4}^{2}=2$ is nicely consistent with the stability conditions mentioned above 3 Concerning the conditions $m_{2.4}^{2}>0$, the class of potentials is large and they can generically be satisfied.

As expected from the non-perturbative canonical analysis, conditions (3) and (4) to have 5 DoFs have led to $m_{0}^{2}=0$, which implies the absence of the ghost sixth mode at the linear level. Needless to say, after the analysis of [15], in these theories five DoF propagate both at linear and at nonlinear level. Notice the important role of the function $\mathcal{C}$, i.e. the integration constant of (5): if $\mathcal{C}=0$ then $m_{1}^{2}=0$ and only $3 \mathrm{DoF}$ propagate at the quadratic level, i.e. the theory is strongly coupled at all scales.

Still at quadratic level, stability of LB theories was studied also in FRW curved spacetime in [14]. For instance in deSitter space with curvature $\mathrm{H}^{2}$, five stable modes propagate provided $\mathrm{H}^{2}<m_{4}^{4} /\left(3\left(m_{4}^{2}-m_{3}^{2}\right)+m_{2}^{2}\right)$. This means that the graviton mass scale has to be larger than the Universe curvature, or in other words that it can take physically interesting values.

Stückelberg Point of View. In any theory with a broken gauge symmetry, the symmetry can be recovered by introducing extra Stückelberg fields. In our case, in the unitary gauge, the potential (9) depends on the rotationally invariant scalars made out of the $3 \mathrm{D}$ matrices $X_{j}^{i} \equiv g^{i k} \delta_{k j}, \Gamma_{j}^{i} \equiv \gamma^{i k} \delta_{k j}$, as well as on the 3D scalar $N$. The breaking of diffeomorphisms can be understood as due to the explicit presence of $N$ and of the external

\footnotetext{
2 The Lorentz-Invariant FP case is recovered for $m_{0}=0, m_{i}=m$.

${ }^{3}$ Note, this relation is modified in the general case $\mathcal{L} \neq 0$, and the stability conditions turn into conditions on $\mathcal{L}$ [18].
}

'frozen' metric $\delta_{i j}$ in the potential, and can be restored by using four Stuckelberg fields $\Phi$ and $\Phi^{i}$, for instance by promoting $X, \Gamma$ and $N$ to manifestly gauge invariant quantities (see [10, 11] for a thorough analysis).

In the standard expansion around Minkowski space, $\Phi=t+\phi^{0}, \Phi^{i}=x^{i}+\phi^{i}$, the metric fluctuations $h_{\mu \nu}$ in the quadratic Lagrangian (12) are replaced by the gauge invariant combinations $H_{\mu \nu}=h_{\mu \nu}-2 \partial_{(\mu} \phi_{\nu)}$. Because $m_{0}^{2}$ vanishes, $\phi^{0}$ does not propagate. On the contrary the three spatial Stückelberg fields do. One can also decompose them in transverse and longitudinal parts, $\phi_{i}=A_{i}+\partial_{i} \varphi$, which makes clear the difference with the known Lorentz-invariant case. Indeed, while in the Lorentz-invariant case the longitudinal field $\varphi$ is made canonical through rescaling by $1 / m^{2} M_{p}$, which leads to low strong coupling scale discontinuity and Vainshtein radius, here all three fields are made canonical by the minimal normalization $\sim 1 / m M_{p}$. In particular for $\varphi$ this fact is due to the difference $m_{1}^{2}-m_{4}^{2}$ being nonzero (see (14) below) and is thus a strict consequence of Lorentz-breaking in the gravitational sector.

Together with the standard canonization of the graviton this normalization leads to three important consequences in the full theory: i) the cutoff of the theory is $\Lambda_{2}=\left(m M_{p l}\right)^{1 / 2}$ [10]; ii) via mixing with the metric, the coupling of the Stückelberg fields to matter sources vanishes as $m$, and thus no discontinuity is present; iii) again in the presence of sources, there is no scale of nonlinearity (Vainshtein) larger than the standard Schwarzschild radius $R_{s}$ (provided $\Lambda_{2}>1 / R_{s}$ ).

It is also instructive to show the quadratic Lagrangian of canonical propagating fields in the 'decoupling limit', defined as $m \rightarrow 0, M_{P l} \rightarrow \infty$ keeping $\Lambda_{2} \equiv \sqrt{m M_{P l}}$ and $T / M_{P l}$ finite. One has symbolically

$$
\begin{aligned}
\mathcal{L}_{\text {eff }}= & h_{c} \square h_{c}+(\dot{\varphi})^{2}-\frac{m_{1}^{2}\left(m_{2}^{2}-m_{3}^{3}\right)}{2 m_{4}^{2}\left(m_{1}^{2}-m_{4}^{2}\right)}(\nabla \varphi)^{2}+ \\
& +\left(\dot{A}_{i}^{c}\right)^{2}-\frac{m_{2}^{2}}{m_{1}^{2}}\left(\nabla_{j} A_{i}^{c}\right)^{2}+\frac{\hat{h}_{c}}{M_{P l}} T
\end{aligned}
$$

which has two transverse tensor DoF in $h_{c}$, two in the transverse vector $A_{i}^{c}$ and one in the scalar $\varphi_{c}$. All these modes are massive, with different masses (they is not visible in the decoupling limit, but see [10]). Note that the scalar kinetic term in (14) is singular in the LI limit, where all $m_{i} \rightarrow m$. Lorentz-breaking is present in the dispersion relations of vector and scalar, which can propagate at speeds different from the one of the light. The presence of these three extra polarizations, in addition to the interesting massive nature of transverse gravitational waves (see e.g. 22]), could thus lead to striking signals already in the forthcoming gravitational waves searches.

Phenomenology. Let us focus on the gravitatiaonal potential generated by a static source, with a non-zero energy-momentum-tensor component $t_{00}$.

At linearized level, the gauge invariant gravitational 
potential $\Phi=h_{00} / 2$ is (we set $M_{P}=1$ here)

$$
\begin{aligned}
\Phi & =\frac{\Delta+m_{2}^{2} \frac{m_{2}^{2}-3 m_{3}^{2}}{m_{2}^{2}-m_{3}^{2}}}{2 \Delta^{2}+\Delta m_{4}^{2} \frac{m_{4}^{2}-4 m_{2}^{2}}{m_{2}^{2}-m_{3}^{2}}+3 m_{4}^{4} \frac{m_{2}^{2}}{m_{2}^{2}-m_{3}^{2}}} t_{00} \\
& \equiv\left[\frac{A_{1}}{\Delta-M_{1}^{2}}+\frac{A_{2}}{\Delta-M_{2}^{2}}\right] \frac{t_{00}}{2},
\end{aligned}
$$

where the squared masses $M_{1,2}^{2} \sim m^{2}$ and the dimensionless coefficients $A_{1,2}$ in the formal decomposition of the second line depend on the mass parameters $m_{i}^{2}$, and $A_{1}+A_{2}=1$ [20]. The squared masses $M_{1,2}^{2}$ may turn out to be real or complex. As a result, the radial profile of the gravitational potential from a point source can be Yukawa, oscillatory, damped (or even plain exponential or Newton, for some special choices of the $m_{i}^{2}$.) Once the squared masses $M_{1}^{2}$ and $M_{2}^{2}$ are real positive, the linearized potential is a sum of two Yukawa terms:

$$
\Phi=\frac{M}{r}\left(A_{1} e^{-M_{1} r}+A_{2} e^{-M_{2} r}\right) .
$$

At small $r$ both gauge invariant potentials 10] reduce to the linearized GR result, i.e. there is no discontinuity.

In the full nonlinear theories that we propose, we can go one step further. In fact, the crucial difference with the Lorentz invariant case is that here we can use perturbation theory. This is actually required to confront with standard post-Newtonian (PN) tests. Using second order perturbation theory, we find that the vacuum spherically symmetric solution can be written, in appropriate Newtonian coordinates $(\tau, \rho)$, in the form

$$
\begin{aligned}
& d s^{2}=-\left[1-\frac{2 M}{\rho}+m^{2} \rho^{2}\left(c_{1} \frac{M}{\rho}+c_{2} \frac{M^{2}}{\rho^{2}} \ln (m \rho)\right)\right] d \tau^{2} \\
& +\rho^{2} d \Omega^{2}+\left[1-\frac{2 M}{\rho}+m^{2} \rho^{2}\left(c_{3} \frac{M}{\rho}+c_{4} \frac{M^{2}}{\rho^{2}}\right)\right]^{-1} d \rho^{2}(17)
\end{aligned}
$$

where for convenience the metric has also been expanded to leading order in $m \rho \ll 1$. The constants $c_{i}$ depend on the chosen potentials $\mathcal{U}$ and $\mathcal{C}$. This result shows that the perturbation expansion near a source has no Vainshtein cutoff, is valid as in GR down to $\rho \sim 2 M$, and is continuous in the $m \rightarrow 0$ limit, also at nonlinear level.

The choice of Newtonian coordinates is allowed because the coupling with matter preserves general covariance, and we also note that it enables us to show that the leading corrections start at $O\left(\mathrm{~m}^{2}\right)$ (unlike what would appear from the expansion of (16) at small $r$, where $g_{\theta \theta}$ and $g_{\varphi \varphi}$ are not in the standard Newtonian form [10]).

In the plain case (16) of large-distance Yukawa falloff, a fairly strong bound on the scale $m$ can be set from the existence of the largest gravitational bound states [21], which nowadays translates into the bound $1 / m \gtrsim 5 \mathrm{Mpc}$, or $m \lesssim 10^{-30} \mathrm{eV}$. This strong bound implies that the proposed models of massive gravity automatically pass the solar system tests, both at Newtonian and at PN level, given the above expressions (17).
As a phenomenological comment, we note that if $M_{1}$ and $M_{2}$ are not of the same scale, e.g. if $M_{1} \gg M_{2}$, and $A_{1}<0$, then at intermediate distances the radial force is enhanced with the respect to the Newtonian case, leading to the flattening of rotation curves also in the absence of dark matter (before the Yukawa falloff at further larger distances). In this case, PN tests may become more relevant. We leave this scenario for a future investigation.

Finally, let us briefly comment on cosmological solutions. In LI massive gravity, Friedmann-RobertsonWalker backgrounds simply do not exist 23, 24]. Viable cosmologies do exist in the bigravity approach 24, 25], where however cosmological perturbations are strongly coupled [26]. In the present models it is likely that some extension will also be needed for cosmology (similar e.g. to [27]). Perturbations may instead turn out to be well-behaved, thanks to the absence of strong nonlinearities. We will address these issues in a separate work [18].

Conclusions. We analyzed the general theories of massive gravity with five propagating DoF and unbroken rotations, which can be candidates for healthy large distance modifications of gravity. The conditions for these theories, formulated in [15], were solved in general, in terms of three free functions. Then, as an example, we described a large class of models that can be analyzed explicitly, and have very interesting features. Namely: (a) Five DoF propagate both at linear and nonlinear level. (b) Gravitational waves are massive, including the three new graviton polarizations. These propagate with Lorentz-breaking dispersion relations, and may lead to striking signals in forthcoming gravitational wave experiments. (c) In static solutions, at short distances $(m r \ll 1)$ the vDVZ discontinuity is absent, and there is no need to rely on nonlinear effects to pass the solar system tests of gravity; at large distances $m r \gg 1$ the gravitational potential is typically screened á la Yukawa, which is the desired behaviour of massive gravity. (d) In this case, one can set a limit on the graviton mass of $m \lesssim 10^{-30} \mathrm{eV}$. (e) As an effective theory the ultraviolet cutoff is $\Lambda_{2}=\sqrt{m M_{\mathrm{pl}}}$, the highest possible in the absence of a fundamental Higgs mechanism for gravity. Because of the above bound, this corresponds to distances of $\sim 10^{-3} \mathrm{~mm}$, below which nonlinear and/or quantum corrections may lead to deviations from GR. This range is of interest for short-distance gravity probes [28]. (f) Aside from this cutoff, the weak field approximation near a macroscopic source of mass $M$ is valid down to the Schwarzschild radius $R_{S}=2 M / M_{P l}^{2}$, as in GR. Thus, a big advantage with respect to the Lorentz-invariant theories is that here modified gravity remains weak as GR in all the phenomenologically interesting regimes.

Of course to seriously compete with GR, massive gravity has to do more than just pass the solar system tests; it has to reproduce the many successes of GR, ranging from emission of gravitational waves from binary systems to CMB temperature fluctuations. Having a calculable weakly coupled theory at hand is of great help, if not a prerequisite, in carrying out the program. 
Finally, we also remark that the conditions for $5 \mathrm{DoF}$, or $m_{0}^{2}=0$ at quadratic level, are not stable under quantum corrections, if one is willing to consider them. Violations of these conditions spoil the counting of DoF, reintroducing the sixth mode, usually at the cutoff scale. This issue is shared among most massive gravity theories, and calls for a proper symmetry protection for the framework described here.
[1] M. Fierz and W. Pauli, Proc. Roy. Soc. Lond. A 173, 211 (1939).

[2] D.G. Boulware and S. Deser, Phys. Lett. B 40, 227 (1972).

[3] C. de Rham, G. Gabadadze, A.J. Tolley, Phys. Rev. Lett. 106, 231101 (2011).

[4] S.F. Hassan and R.A. Rosen, Phys. Rev. Lett. 108, 041101 (2012); S.F. Hassan, R.A. Rosen and A. SchmidtMay, JHEP 1202, 026 (2012) ; S.F. Hassan and R.A. Rosen, JHEP 1204 (2012) 123

[5] C. de Rham, G. Gabadadze and A. Tolley, arXiv:1107.3820 [hep-th].

[6] H. van Dam and M.J.G. Veltman, Nucl. Phys. B 22 (1970) 397; Y. Iwasaki, Phys. Rev. D 2 (1970) 2255; V.I.Zakharov, JETP Lett. 12 (1971) 198.

[7] A.I. Vainshtein, Phys. Lett. B 39, 393 (1972);

[8] E. Babichev, C. Deffayet and R. Ziour, Phys. Rev. Lett. 103, 201102 (2009); N. Kaloper, A. Padilla and N. Tanahashi, JHEP 1110, 148 (2011); G. Chkareuli and D. Pirtskhalava, Phys. Lett. B 713, 99 (2012).

[9] G. Dvali, New J. Phys. 8 (2006) 326 hep-th/0610013.

[10] V.A. Rubakov, arXiv:hep-th/0407104.

[11] S.L. Dubovsky, JHEP 0410, 076 (2004); V.A. Rubakov and P.G. Tinyakov, Phys. Usp. 51, 759 (2008).

[12] G. Gabadadze and L. Grisa, Phys. Lett. B 617 (2005) 124; L. Grisa, JHEP 0811 (2008) 023.

[13] Z. Berezhiani, D. Comelli, F. Nesti and L. Pilo, Phys. Rev. Lett. 99, 131101 (2007).

[14] D. Blas, D. Comelli, F. Nesti, L. Pilo, Phys. Rev. D80, 044025 (2009).

[15] D. Comelli, M. Crisostomi, F. Nesti and L. Pilo, Phys.
Rev. D 86, 101502 (2012).

[16] R.L. Arnowitt, S. Deser and C.W. Misner, gr-qc/0405109

[17] N. Arkani-Hamed, H. Georgi and M.D. Schwartz, Annals Phys. 305, 96 (2003).

[18] D. Comelli, F. Nesti, L. Pilo, to appear.

[19] D.B. Fairlie and A.N. Leznov, Journal of Geometry and Physics 16 (1995) 385-390.

[20] Z. Berezhiani, F. Nesti, L. Pilo and N. Rossi, JHEP 0907, 083 (2009) .

[21] A. S. Goldhaber and M. M. Nieto, Phys. Rev. D 9 (1974) 1119.

[22] A. E. Gumrukcuoglu, S. Kuroyanagi, C. Lin, S. Mukohyama and N. Tanahashi, Class. Quant. Grav. 29 (2012) 235026.

[23] G. D'Amico, C. de Rham, S. Dubovsky, G. Gabadadze, D. Pirtskhalava and A. J. Tolley, Phys. Rev. D 84, 124046 (2011).

[24] D. Comelli, M. Crisostomi, F. Nesti and L. Pilo JHEP 1203, 067 (2012) [Erratum-ibid. 1206, 020 (2012)]. M. von Strauss, A. Schmidt-May, J. Enander, E. Mortsell and S.F. Hassan, JCAP 1203, 042 (2012); M.S. Volkov, JHEP 1201, 035 (2012).

[25] M. S. Volkov, Phys. Rev. D 86 (2012) 061502.

[26] D. Comelli, M. Crisostomi and L. Pilo, JHEP 1206, 085 (2012) .

[27] G. D'Amico, G. Gabadadze, L. Hui and D. Pirtskhalava, arXiv:1206.4253 [hep-th].

[28] E. G. Adelberger, J. H. Gundlach, B. R. Heckel, S. Hoedl and S. Schlamminger, Prog. Part. Nucl. Phys. 62 (2009) 102. 